\title{
A Single-Axis Low-Cost Accelerometer Fabricated Using Printed-Circuit-Board Techniques
}

\author{
Dapeng Qiao, Grantham K. H. Pang, Senior Member, IEEE, Man-Kit Mui, and \\ David C. C. Lam, Senior Member, IEEE
}

\begin{abstract}
This letter presents a new single-axis low-cost printed-circuit-board (PCB)-based accelerometer. Motivated by the need to develop low-cost human-sensing accelerometer, a capacitive PCB-based microelectromechanical-system (MEMS) accelerometer is developed, and the experimental results of the prototype have demonstrated that the objective is feasible. The PCB-based MEMS accelerometer is fabricated with a metal film adhered to a PCB, which forms the two electrodes of the sensing capacitor of the accelerometer. This signal is compared with the output signal of a commercially available accelerometer, ADXL330, by Analog Devices. The prototypes are designed to have a dynamic range of $\pm 5 \mathrm{~g}$ and a bandwidth from 0 to $20 \mathrm{~Hz}$, which covers the range of accelerations for human limbs. The result shows that the new low-cost accelerometer can fulfill the requirement of human motion sensing.
\end{abstract}

Index Terms-Accelerometer, human motion sensing, wireless game controller.

\section{INTRODUCTION}

$\mathbf{T}$ HE COST and size of accelerometers have been reduced with advances in microelectromechanical-system (MEMS) technology. Wide applications have been found in inertial-navigation system, hard-disk-drive, head-positioning control system, virtual-reality system, automobile control system, and smart phones. Accelerometers are also used in sophisticated remote controls in gaming systems like Wii Remote [1]. Despite drops in prices, accelerometers are still considered expensive when used together with the custom-designed chips and interface electronics, and new classes of low-cost accelerometers are needed to enable popular adoption in consumer products and gaming devices. To drive down cost, accelerometer designed and fabricated using low-cost PCB processes and materials are explored and compared with the ADXL330 by Analog Devices [2], which is used to sense the player's hand position in three dimensions and is a MEMS silicon accelerometer.

When accelerometers are used in toys, remote controls for video game, or applications in human limbs, the requirements of motion sensing are varied for different parts of human limbs.

Manuscript received August 26, 2009; revised September 24, 2009. First published November 3, 2009; current version published November 20, 2009. The review of this letter was arranged by Editor C.-P. Chang.

D. Qiao and G. K. H. Pang are with the Industrial Automation Research Laboratory, Department of Electrical and Electronic Engineering, The University of Hong Kong, Hong Kong (e-mail: dpqiao@eee.hku.hk; gpang@eee.hku.hk).

M.-K. Mui and D. C. C. Lam are with the Department of Mechanical Engineering, Hong Kong University of Science and Technology, Kowloon, Hong Kong (e-mail: muimk@ hotmail.com; medcclam@ust.hk).

Color versions of one or more of the figures in this letter are available online at http://ieeexplore.ieee.org.

Digital Object Identifier 10.1109/LED.2009.2033720
TABLE I

SUMMARY OF ACCELERATION FREQUENCY AND RANGE OF HUMAN MOTIONS

\begin{tabular}{|l|l|l|}
\hline Device type & Acceleration & Frequency range \\
\hline Head devices & $0.5 \mathrm{~g}$ to $9 \mathrm{~g}$ & $3.5 \mathrm{~Hz}$ to $8 \mathrm{~Hz}$ \\
\hline $\begin{array}{l}\text { Upper-body devices } \\
\text { (hand, arm) }\end{array}$ & $0.5 \mathrm{~g}$ to $9 \mathrm{~g}$ & less than $12 \mathrm{~Hz}$ \\
\hline Hand/finger devices & $0.04 \mathrm{~g}$ to $1 \mathrm{~g}$ & $8 \mathrm{~Hz}$ to $12 \mathrm{~Hz}$ \\
\hline Foot-leg devices & 0.2 to $6.6 \mathrm{~g}$ & less than $12 \mathrm{~Hz}$ \\
\hline
\end{tabular}

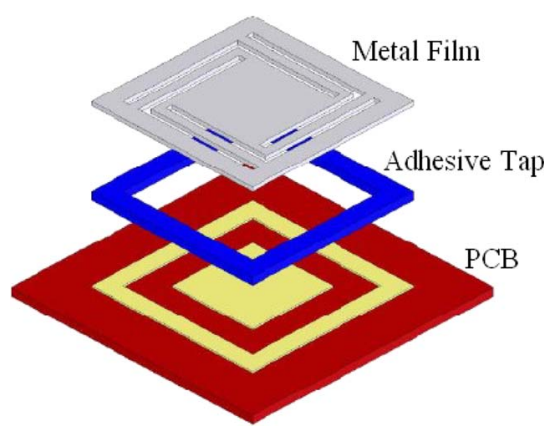

Fig. 1. Structure of the sensor.

A summary of the acceleration and frequency range of human motions is shown in Table I [3], [4].

Recently, a MEMS capacitive-type accelerometer fabricated using printed-circuit processing techniques is proposed [5]. The author has stated the advantages of PCB MEMS as low cost, ease of integration with electronics, and suitability for highvolume manufacturing and large-area applications. In this letter, a PCB-based accelerometer which could meet the humanmotion-sensing requirements is presented. It can be used as a substitute for costly silicon accelerometer.

\section{ACCelerometer Sensor Design}

The design specification of the PCB accelerometer sensor is defined based on the human-motion-sensing requirements. As most of the activities of the arm are less than $5 \mathrm{~g}, \pm 5 \mathrm{~g}$ will be set as the target measurement range of the PCB prototype. The bandwidth of the sensor must cover the frequency of arm motion, which is $12 \mathrm{~Hz}$.

The size of the accelerometer is the other design issue to be considered. It should be minimized in order to reduce the footprint occupied by the sensor and the cost of material. The prototype discussed in this letter is a capacitance type of accelerometer, which consists of a proof mass, an adhesive tape, and a piece of PCB, as shown in Fig. 1. A piece of metal film, which is etched into a center plate with suspension connected to 


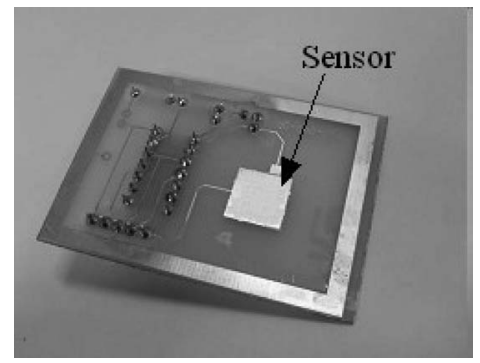

Fig. 2. Sensor prototype.

a frame, is adhesively attached to the PCB by the adhesive tape. The metal film and the etched copper pattern on the PCB facing the metal plate forms the two capacitor electrodes separated by a gas film. When accelerated, the proof mass moves, and the gap distance changes with acceleration, which causes change of the capacitance of the sensor. The change in the capacitance value would be converted to a voltage output by the interface electronic.

The design of the sensor should consider two questions affecting the behavior of the sensor: the sensitivity and the damping of the sensor. The sensitivity of the sensor is dependent on the weight of the proof mass and the stiffness of the structure suspending the proof mass. The metal film adhesively attached to the PCB should have a suitable structure (including the shape and thickness) to effectively deform according to different accelerations, while the material of the metal should be carefully selected because it also determines the sensitivity of the sensor. The design is conducted based on a number of experiments with the simulation of the software ANSYS. Many groups of dimension value and materials are tested to obtain the best sensitivity and less damping. The capacitance of the sensor can be calculated to correspond to the structure dimension, the stiffness of the sensor, and the thickness of the adhesive tape. At last, the dimension of the sensor is determined, the outline of which is around $10 \mathrm{~mm} \times 10 \mathrm{~mm}$ with a sensitivity of $0.103 \mathrm{pf} / \mathrm{g}$ (more details on the fabrication of the sensor can be found in [6] and [7]).

The production of the sensor is mainly through the etching of a metal film. The minimum value of the dimension is about $50 \mu \mathrm{m}$, which means the dimension can be accurately drawn by the general photolithography used in PCB making. Therefore, the production of the sensor is easy and low cost. Fig. 2 shows the photograph of the sensor prototype. From this figure, the direction of the two conductive wires connected to the two electrodes of the sensor is carefully designed to avoid the parasitic effect.

\section{PCB-BAsed AcCelerometer Readout Circuit}

In the experimental setup, the sensor was connected to the readout circuit which produced an analog voltage signal proportional to the capacitance of the sensor, which requires high capacitance sensitivity. The block diagram of this circuit is shown in Fig. 3.

The whole readout circuit consists of sensor interface circuit, amplification circuit, rectifier, low-pass filter, and zero-setting circuit. The sensor interface circuit is printed on the PCB beside the sensor. The sensor interface circuit shown in Fig. 4 reads the

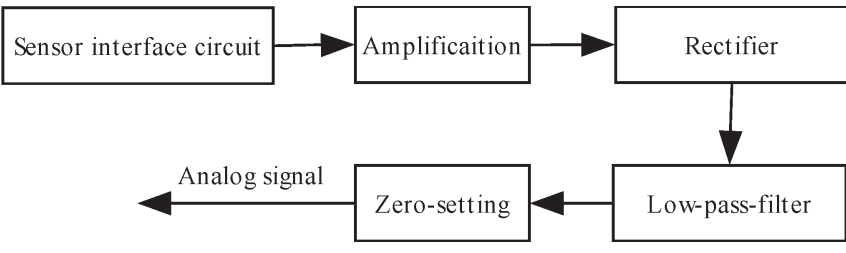

Fig. 3. Block diagram of circuit for analog voltage signal proportional to the sensor's capacitance.

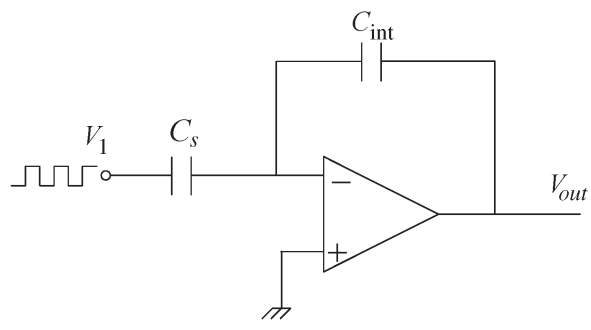

Fig. 4. Schematic diagram of sensor interface circuit.

TABLE II

SPECIFICATIONS OF THE SENSOR

\begin{tabular}{|c|c|c|}
\hline Sensitivity & Bandwidth & Dynamic range* \\
\hline $0.3 \mathrm{~V} / \mathrm{g}$ & $16 \mathrm{~Hz}$ & $-5 \mathrm{~g}$ to $5 \mathrm{~g}$ \\
\hline
\end{tabular}
*The dynamic range of the ADXL330 is also $-5 \mathrm{~g}$ to $5 \mathrm{~g}$. In this experiment,
the readout circuit is designed to measure $-5 \mathrm{~g}$ to $5 \mathrm{~g}$, with a sensitivity of
$0.3 \mathrm{~V} / \mathrm{g}$.

capacitance value of the sensor and transforms it into an analog signal.

In the discussed circuit, the sensor capacitor is charged with digital pulse, and the output signal which is proportional to the sensor's capacitance is integrated

$$
V_{\text {out }}=V_{1} \frac{C_{s}}{C_{\text {int }}} .
$$

In Fig. 4, the signal $V_{\text {out }}$ is sent to an amplification circuit to get a suitable amplitude to be rectified in the next step. After amplification, $V_{\text {out }}$ is rectified into a dc voltage which is nearly proportional to the amplitude of $V_{\text {out }}$, and then, the high-frequency part is filtered out. The low-pass filter is used to filter out the high-frequency part, which is the noise of the circuit, and the $50 \mathrm{~Hz}$ from the mains. At the end of the readout circuit, the output signal is given an offset, which means that when the acceleration is zero, the sensor's capacitance would correspond to a certain voltage value.

The source wave $V_{1}$ for the sensor was a $5-\mathrm{kHz}$ square wave which had a maximum of $0.5 \mathrm{~V}$ and minimum of $-0.5 \mathrm{~V}$. This square wave was produced by a waveform generator, and a dc power supply served as the power source of the readout circuit. The analog voltage of the readout circuit was read by oscilloscope. The specifications of the sensor with the readout circuit are shown in Table II.

\section{EXPERIMENTS AND ANALYSIS}

A three-axis silicon accelerometer from Analog Device (ADXL 330) [8] with analog voltage output was used as a reference device, and only one axis was used to compare with the output signal of the PCB-based accelerometer. An evaluation board (EVAL-ADXL330Z) provided by Analog Devices was used to obtain an output voltage from ADXL330. 


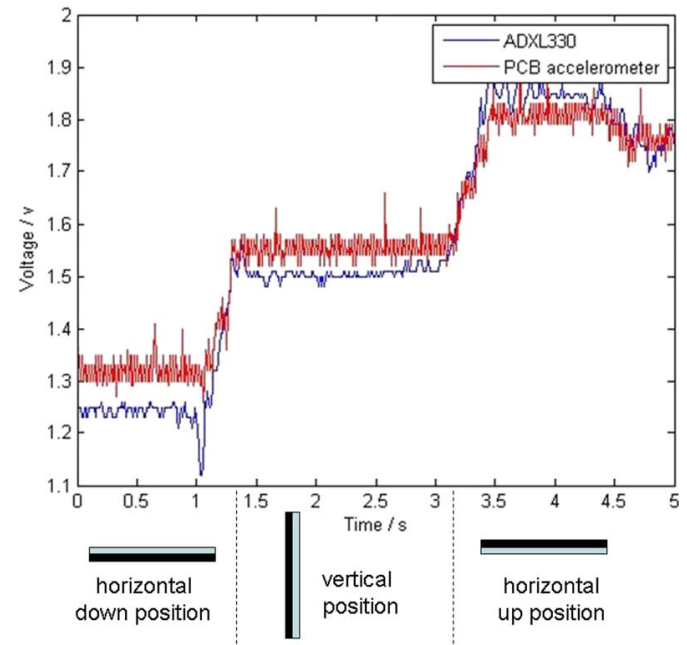

Fig. 5. Static acceleration responses of PCB-based accelerometer compared with the response of ADXL330.

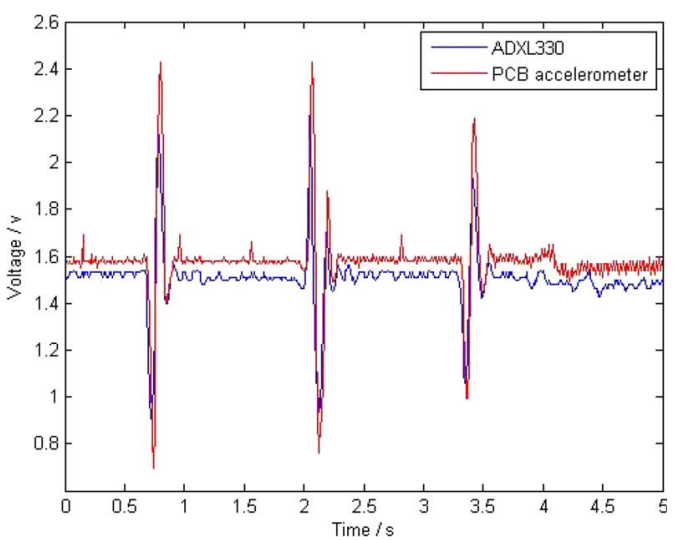

Fig. 6. Abrupt-shake responses.

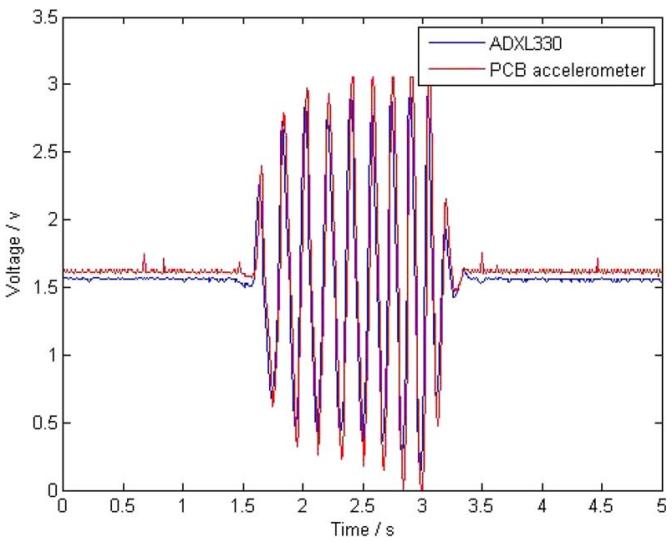

Fig. 7. Quick-shake responses.

To compare the output of these two accelerometers under identical acceleration, they were affixed onto the same substrate in the experiment. Then, the oscilloscope measured the outputs of the two accelerometers which were under the same acceleration. The results are shown in Figs. 5-7.

In Fig. 5, the output of the two accelerometers under static acceleration is shown. From time $0-1.3 \mathrm{~s}$, the two accelerometers were hand held in a horizontal down position. Then, the two devices were placed in a vertical position until $3.2 \mathrm{~s}$ was reached. Afterward, the two devices were flipped to a horizontal up position. It can be seen that the waveforms are nearly the same. Both have a response of $\sim 0.3 \mathrm{~V}$ for $1 \mathrm{~g}$ of change, which is close to the designed specification. The absolute difference between the two measurements has been calculated, and the average of this difference in $5 \mathrm{~s}$ is $0.05 \mathrm{~V}$, which is very small.

In a second evaluation, the two accelerometers were shaken suddenly three times, and their responses to abrupt shaking are shown in Fig. 6.

The first shake occurred at around $0.7 \mathrm{~s}$ and the two accelerometers moved from left to right and then suddenly stopped, while the other two shakes took place at 2.1 and $3.4 \mathrm{~s}$, respectively. These motions are clearly detected and are shown in the figure. Similar waveforms by both the PCB-based accelerometer and ADXL330 have been recorded. The average absolute difference is $0.08 \mathrm{~V}$.

Fig. 7 shows the quick-shake responses which stand for the higher frequency response, and again, the waveform of PCB accelerometer is essentially identical to that of ADXL330. The shake's frequency is about $8 \mathrm{~Hz}$, which is near the maximum of usual arm movement. The average absolute difference is $0.15 \mathrm{~V}$. This value can be decreased by modifying the offset of the circuit output.

\section{CONCLUSION}

A low-cost PCB-based accelerometer has been designed and fabricated. The performance of the PCB-based accelerometer is shown to be comparable to a commercial accelerometer (ADXL330) in human motion sensing. With a simple structure and no custom-design chips, we can expect that this approach could possibly result in a lower cost accelerometer than equivalent devices manufactured by Si micromachining. Consequently, the expected ratio of performance over price will be highly attractive. This will enable more PCB-based accelerometers to be adopted in toys, biomedical and navigation applications that have low measure range, and low-temperature requirements with no strict limits on structure size.

For future work, more evaluations on the PCB-based MEMS accelerometer would be carried out. The thermal stability of the accelerometer needs to be examined. Other important issues such as rigidity, fatigue, drift, and swelling on the material properties would also have to be studied.

\section{REFERENCES}

[1] [Online]. Available: http://en.wikipedia.org/wiki/Wii_Remote

[2] [Online]. Available: http://www.analog.com/en/prod/0,2877,ADXL330,00. html

[3] C. Verplaetse, "Inertial proprioceptive devices: Self-motion-sensing toys and tools," IBM Syst. J., vol. 35, no. 3/4, pp. 639-650, 1996.

[4] J. Yang, W. Chang, W. C. Bang, E. S. Choi, K. H. Kang, and S. J. Cho, "Analysis and compensation of errors in the input device based on inertial sensors," in Proc. Int. Conf. Inf. Technol., Coding Comput., 2004, vol. 2, pp. 790-796.

[5] J. E. Rogers, R. Ramadoss, P. M. Ozmun, and R. N. Dean, "MEMS accelerometer fabricated using printed circuit processing techniques," in Proc. IEEE Int. Symp. Ind. Electron., Jun. 4-7, 2007, pp. 3250-3254.

[6] M. K. Mui and D. C. C. Lam, "Development and characterizations of low cost accelerometers," in Proc. Int. Conf. EMAP, Dec. 11-14, 2006, pp. 1-5.

[7] M. K. Mui and D. C. C. Lam, "Design-process modeling of meso-accelerometers," IEEE Trans. Electron. Packag. Manuf., 2009, to be published.

[8] [Online]. Available: http://www.analog.com/static/imported-files/data_ sheets/ADXL330.pdf 